\section{Reverse-link performance of synchronous DS-CDMA systems in dispersive Rician multipath fading channels}

\author{
S.H. Hwang and L. Hanzo
}

\begin{abstract}
The reverse-link performance of synchronous DS-CDMA is investigated in Rician multipath fading environments. The numerical results show that the performance benefits of synchronous transmission depend on the specular path's power as well as on the multipath intensity decay factor of the channel. The achievable bit-error-rate reduction may become as high as an order of magnitude in comparison to the corresponding asynchronous system.
\end{abstract}

Introduction: Synchronous direct-sequence code-division multipleaccess (DS-CDMA) uplink transmission has been proposed for reducing the effects of multiple access interference (MAI) [1, 2], with the additional benefit of having a lower multi-user detection, or interference cancellation complexity, than asynchronous systems [3]. Uplink synchronous DS-CDMA is therefore considered an attractive technology for future mobile communication systems [4] or mobile broadband wireless access [5]. For such applications, uplink synchronous DS-CDMA requires accurate adaptive timing advance control, which is readily achievable in low-mobility indoor and/or pedestrian environments. However, previous studies $[1,2]$ have assumed the presence of Rayleigh fading and have neglected the performance benefit of having a specular component. In reality, the indoor channel often exhibits a strong line-of-sight (LOS) component [6], and therefore this Letter presents the analysis of the scenario of a direct LOS path, which results in Rician multipath fading.

System model and analysis: The synchronous DS-CDMA uplink described in [1] is considered, in which an orthogonal spreading sequence is used. In the synchronous uplink, the arrival time of the first Rake receiver branch is assumed to be synchronous but the remaining branches are asynchronous, because this can be readily achieved by state-of-the-art synchronisation techniques [3]. The Rayleigh fading channel model is modified by adding a known and constant LOS component, namely $\alpha e^{j \phi_{\alpha}}$, to the first tap of the tapped delay-line representation of the channel. The first path of this model exhibits Rician fading [7]. Two different multipath intensity profiles (MIP) are considered here, the equal-weight MIP of $E\left[\left\{\beta_{l}^{(k)}\right\}^{2}\right]=$ $1 / L^{(k)}, l=0, l=0, \ldots, L^{(k)}-1$, and the negative exponentially decaying MIP of $E\left[\left\{\beta_{l}^{(k)}\right\}^{2}\right]=\Omega_{0}^{(k)} e^{-\delta}$, for $\delta>0$, where $\beta_{l}^{(k)}$ refers to the Rayleigh distributed envelope of the $l$ th faded path of the $k$ th user, $\Omega_{0}^{(k)}$ is the power associated with the first faded path, $L^{(k)}$ is the total number of resolvable paths, and $\delta$ represents the decay factor of the average path power as a function of the path delay. Perfect power control is assumed.

The Rake receiver finger associated with the first resolvable path is assumed to track only the specular path, whereas the Rayleigh faded multipath components simply contribute to the interference [7]. The final received waveform consists of a signal component, a white noise component, a multipath interference and an MAI component, which may be assumed to be Gaussian, when the number of users, $K$, is high [8]. Extending the derivations of [2], the MAI terms of both the first as well as the other branches are modelled as Gaussian processes having variances equal to the MAI variances for both $n=0$ and $n \geq 1$. Thus the final decision variable, $U$, conditioned on the fading envelopes of the desired user, $\left\{\beta_{n}^{(1)}\right\}$, can be shown to be a Gaussian random variable having a mean of $U_{s}=\sqrt{ }\left(E_{b} T / 2\right)\left(\alpha^{2}+\sum_{n=1}^{L_{r}-1}\left\{\beta_{n}^{(1)}\right\}^{2}\right)$, and variance of:

$$
\begin{aligned}
\sigma_{T}^{2}= & \frac{E_{b} T(2 N-3)}{12 N(N-1)} \alpha^{2} \sum_{k=2}^{K} \sum_{l=1}^{L^{(k)}-1} \Omega_{l}^{(k)}+\frac{E_{b} T(N-1)}{6 N^{2}} \\
& \times \sum_{n=1}^{L_{r}-1}\left\{\beta_{n}^{(1)}\right\}^{2} \sum_{k=2}^{K}\left(\alpha^{2}+\sum_{l=1}^{L^{(k)}-1} \Omega_{l}^{(k)}\right) \\
& +\frac{E_{b} T}{4 N}\left(\alpha^{2}+\sum_{n=1}^{L_{r}-1}\left\{\beta_{n}^{(1)}\right\}^{2}\right) \sum_{l=1}^{L^{(k)}-1} \Omega_{l}^{(1)} \\
& +\frac{T \eta_{0}}{4}\left(\alpha^{2}+\sum_{n=1}^{L_{r}-1}\left\{\beta_{n}^{(1)}\right\}^{2}\right)
\end{aligned}
$$

where $L_{r}$ is the number of branches in the Rake receiver, $E_{b}$ is the average transmitted energy per bit, $T$ is the data bit duration, $N$ is the processing gain and $\Omega_{l}^{(k)}=E\left[\left\{\beta_{l}^{(k)}\right\}^{2}\right]$.

Probability of error for equal weight MIP: We assume that the fading statistics for each user are identical, hence the average received power of each resolvable path is the same for all users, yielding $E\left[\left(\beta_{l}^{(k)}\right)^{2}\right]=E\left[\beta_{l}^{2}\right]$. Since binary phase shift keying is used in this analysis, we can invoke the well known result for the conditional probability of error, when it is conditioned on the fading statistics, which is given by:

$$
\operatorname{Pr}\left(\operatorname{error} \mid\left\{\beta_{n}^{(1)}\right\}\right)=Q(\sqrt{\gamma})
$$

where $Q(y)=1 / \sqrt{ }(2 \pi) \int_{y}^{\infty} \exp \left(-x^{2} / 2\right) d x$ and

$$
\begin{aligned}
\gamma= & \frac{u_{s}^{2}}{\sigma_{T}^{2}}=\left[\frac{(N-1)(K-1)\left(\alpha^{2} / \Omega_{0}+\left[q\left(L^{(k)}, \delta\right)-1\right]\right)}{3 N^{2}}\right. \\
& \times \frac{\sum_{n=1}^{L_{r}-1}\left\{\beta_{n}^{(1)}\right\}^{2}}{\alpha_{r}-1}+\frac{(2 N-3)(K-1) \alpha^{2}\left[q\left(L^{(k)}, \delta\right)-1\right]}{6 N(N-1) \alpha^{2}+\sum_{n=1}^{L_{r}-1}\left\{\beta_{n}^{(1)}\right\}^{2}} \\
& \alpha^{2}+\sum_{n=1}^{L_{n}}\left\{\beta_{n}^{(1)}\right\}^{2} \\
& \left.+\frac{\left[q\left(L^{(k)}, \delta\right)-1\right]}{2 N}+\frac{\eta_{0}}{2 E_{b} \Omega_{0}}\right]^{-1} \frac{\alpha^{2}+\sum_{n=1}^{L_{r}-1}\left\{\beta_{n}^{(1)}\right\}^{2}}{\Omega_{0}}
\end{aligned}
$$

where $q\left(L^{(k)}, \delta\right)=\sum_{l=0}^{L^{(k)}-1} e^{-l \delta}=\left(1-e^{L^{(k)}} \delta\right) /\left(1-e^{-\delta}\right)$ and $\Omega_{0}=E\left[\beta_{0}^{2}\right]$.

For $L_{r}>1$, since the path envelopes $\left\{\beta_{n}^{(1)}\right\}$ are independent and Rayleigh distributed, $X=\sum_{n=1}^{L_{r}-1}\left\{\beta_{n}^{(1)}\right\}^{2}$ has a chi-squared distribution with $2\left(L_{r}-1\right)$ degrees of freedom. Using standard integration techniques for removing the conditioning on $\left\{\beta_{n}^{(1)}\right\}$, the final expression for the probability of error becomes:

$$
\operatorname{Pr}(\text { error })=\int_{0}^{\infty} Q(\sqrt{\gamma}) \frac{x^{L_{r}-2}}{\Omega_{0}^{L_{r}-1}\left(L_{r}-2\right) !} e^{-x / \Omega_{0}} d x
$$

Probability of error for negative exponentially decaying MIP: For the case of different average path powers, averaging the conditional probability of error over the density function of $X$ gives the following:

$$
\operatorname{Pr}(\text { error })=\int_{0}^{\infty} Q(\sqrt{\gamma}) \sum_{k=1}^{L_{r}-1} \frac{\pi_{k}}{\Omega_{k}} e^{-x / \Omega_{k}} d x
$$

where $\pi_{k}=\prod_{i=1 ; i \neq k}^{L_{r}-1} x_{k} /\left(x_{k}-x_{i}\right)=\prod_{i=1 ; i \neq k}^{L_{r}-1} \Omega_{k} /\left(\Omega_{k}-\Omega_{i}\right)$.

Results and discussion: In the following, $\alpha^{2} / \Omega_{0}$ is the ratio of the specular power to the average power for the first scattered path. Indoor radio channel measurements show that Rician distributions tend to have a $K$-factor ranging from 2 to $7 \mathrm{~dB}$ [7]. Figs. 1-3 show the average bit-error-rate probabilities against the number of users in conjunction with various numbers of Rake receiver branches, assuming that the total number of paths is five and $N=128$. The bit-errorrate analysis for an asynchronous CDMA system using Rake reception in Rician multipath fading can be found in [9]. For a fixed probability of error, the number of users inferred from the graphs represents the achievable upper bound system capacity. Comparing Figs. 1 and 2 characterises the effect of increasing the MIP decay factor from $\delta=0$ to $\delta=-3 \mathrm{~dB}$. Intuitively, as the parameter $\delta$ increases, the received power of the main path including the nonfading LOS component increases, resulting in a higher specular signalto-noise ratio (SNR). This significantly enhances the achievable performance of the synchronous system. However, when a stronger direct LOS path power is encountered in the context of an exponentially decaying MIP, the achievable performance reflected in Fig. 3 suggests that most systems experience improved BER, when $\alpha^{2} / \Omega_{0}$ increases from one to two. This Figure indicates that the synchronous reverse-link system outperforms the corresponding asynchronous system. As the specular path power and the parameter $\delta$ increase, the effect of all the other paths, except for the initial path, becomes negligible as a result of the higher specular SNR and as a consequence of the reduced diversity gain. 


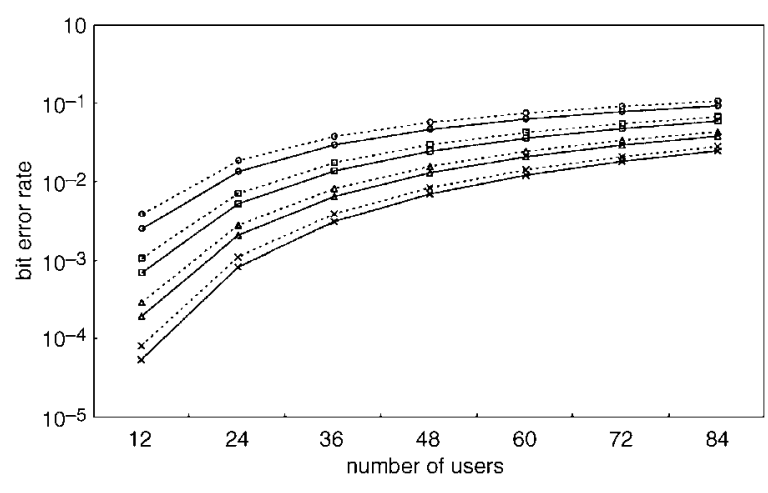

Fig. 1 Bit error rate against number of users for equal-weight MIP $E_{b} \Omega_{0} / \eta_{0}=10 \mathrm{~dB}, \alpha^{2} / \Omega_{0}=0 \mathrm{~dB}, \delta=0.0$

$$
\begin{array}{ll}
\bigcirc L_{r}=2 & \\
\triangle L_{r}=4 & \square L_{r}=3 \\
& \times L_{r}=5
\end{array}
$$

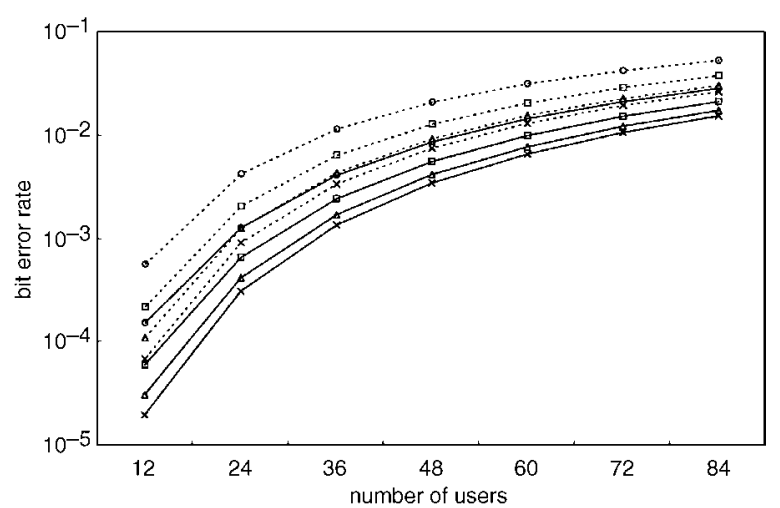

Fig. 2 Bit error rate against number of users for negative exponentially decaying $M I P$

$$
\begin{array}{ll}
E_{b} \Omega_{0} / \eta_{0}=10 \mathrm{~dB}, \alpha^{2} / \Omega_{0}=0 \mathrm{~dB}, \delta=-3 \mathrm{~dB} \\
\hline L_{r}=2 & \cdots \cdot \text { async } \\
\triangle L_{r}=4 & \times L_{r}=3 \\
& \times L_{r}=5
\end{array}
$$

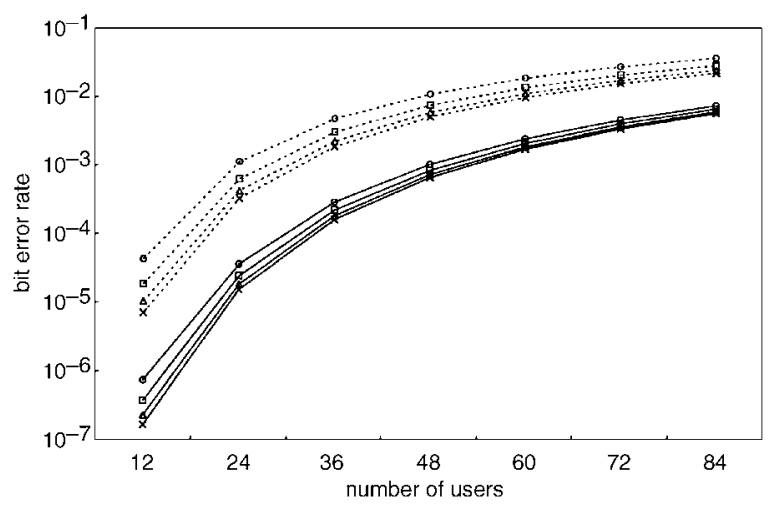

Fig. 3 Bit error rate against number of users for negative exponentially decaying $M I P$

$$
\begin{array}{ll}
E_{b} \Omega_{0} / \eta_{0}=10 \mathrm{~dB}, \alpha^{2} / \Omega_{0}=3 \mathrm{~dB}, \delta=-3 \mathrm{~dB} \\
\hline L_{r}=2 & \quad \cdots \cdots \text { async } \\
\triangle L_{r}=4 & \times L_{r}=3 \\
& \times L_{r}=5
\end{array}
$$

Conclusions: The synchronous reverse-link CDMA system outperforms the corresponding asynchronous system for all the cases considered here. The addition of a direct LOS path component to the channel model increases the performance differences between the synchronous and asynchronous reverse-link systems. Furthermore, the exponential MIP decay factor, $\delta$, has a substantial effect on the achievable system performance in a Rician fading channel. Therefore, the BER improvement achieved with the advent of synchronous transmissions depends on the specular path's power as well as on the MIP decay factor of the channel, since the effect of all the other paths, except for the initial LOS path, becomes negligible as a result of the higher specular SNR and as a consequence of the reduced diversity gain. All in all, as seen in Fig. 3, for the case of a strong direct LOS path or for a high MIP decay factor, the employment of a coherently detected synchronous reverse link has the potential of improving the achievable BER by an order of magnitude.

(C) IEE 2003

Electronics Letters Online No: 20031014

4 August 2003

DOI: 10.1049/el:20031014

S.H. Hwang and L. Hanzo (Department of Electronics and Computer Science, University of Southampton, Highfield, Southampton, SO17 1BJ, United Kingdom)

\section{References}

1 HONG, E.K., et al.: 'Synchronous transmission technique for the reverse link in DS-CDMA terrestrial mobile systems', IEEE Trans. Commun., 1999, 47, (11), pp. 1632-1635

2 HWANG, S.-H., and KIM, D.K.: 'Performance of reverse-link synchronous DS-CDMA system on a frequency-selective multipath fading channel with imperfect power control', EURASIP J. Appl. Signal Process., 2002, (8), pp. 797-806

3 HANZO, L., et al.: 'Single- and multicarrier CDMA' (John Wiley \& IEEE Press, 2003)

4 3GPP TR25.854: Uplink Synchronous Transmission Scheme, May 2001 (ftp://ftp.3gpp.org/)

5 IEEE 802.20 C802.20-03/29: Smart antenna and MC-SCDMA, April 2003

6 RAPPAPORT, T.S.: 'Wireless communications' (Prentice Hall, 1996)

7 WANG, J., and MILSTEIN, L.B.: 'CDMA overlay situations for microcellular mobile communications', IEEE Trans. Commun., 1995, 43, (2/3/4), pp. 603-614

8 VITERBI, A.J.: 'CDMA-principles of spread spectrum communications' (Addison Wesley, 1995)

9 FOERSTER, J.R., and MILSTEIN, L.B.: 'Analysis of hybrid, coherent FDMA/CDMA systems in Ricean multipath fading', IEEE Trans. Commun., 1997, 45, pp. 15-18 